\title{
Surgical Access to the Ovine Fetus the Left Flank Approach
}

\author{
Pringle $\mathrm{KC}^{1 *}$, Seki $\mathrm{Y}^{2}$ and Kitagawa $\mathrm{H}^{2}$ \\ ${ }^{1}$ Department of Obstetrics and Gynaecology, University of \\ Otago, Wellington, New Zealand \\ ${ }^{2}$ Division of Pediatric Surgery, St Marianna University \\ School of Medicine, J apan \\ *Corresponding author: Pringle KC, Professor \\ of Paediatric Surgery, Department of Obstetrics and \\ Gynaecology, University of Otago, Wellington, New \\ Zealand
}

Received: September 06, 2017; Accepted: November 07, 2017; Published: November 15, 2017

\begin{abstract}
Introduction: Most researchers using ovine fetal models use an infraumbilical midline incision. This approach is simple, but has a significant incidence of maternal complications, most of which are not reported. This paper presents a left flank approach, which is less painful and causes few complications.
\end{abstract}

Materials and Methods: The senior author developed this approach, with a Veterinary colleague, during the development of a fetal lamb model for the creation and repair of a diaphragmatic hernia. The evolution of this approach and the changing anaesthetic techniques are presented.

Results and Discussion: This approach has minimal complications. Wound infections are rare, although sterile seromata are common. Blood loss is minimal. The ewes are usually up and eating normally within an hour of completing the procedure.

Conclusion: This approach is slightly more technically demanding. However, the low morbidity makes it an excellent approach to the fetal lamb.

Keywords: Fetal lambs; Fetal surgery; Ovine models

\section{Abbreviations}

L: Left; R: Right; lab: laboratory; DH: Diaphragmatic Hernia; OU: Obstructive Uropathy; BOO: Bladder Outlet Obstruction; U/S: Ultrasound; ETT: Endotracheal Tube; OG: Orogastric; IV: Intravenous

\section{Introduction}

From the very early fetal lamb models [1-5] researchers have utilised a midline infra-umbilical or paramedian incision. A rare exception was Burrington, who explored fetal wound healing [6] and used a Right (R) muscle-splitting incision. Ventral incisions in ewes have a significant morbidity, almost none of which is reported. A rare exception to this statement is the report by Schinckel and Ferguson [1], who reported that 6 of 9 ewes operated on through a paramedian incision had incisional herniae at term. Wound infections are common and the senior author has personal experience of repairing a massive incisional hernia in a ewe who had previously been used for fetal lamb cannulation experiments by another researcher in an era when it was acceptable to use experimental animals for more than 1 experiment. The hernia was, in fact, repaired through the left flank incision described below.

\section{Materials and Methods}

\section{Breeding}

Ewes bred specifically for research are purchased from the AgResearch farm at Kaitoke, about 1 hour's drive north of Wellington. If necessary the ewes are synchronised by insertion of Controlled Internal Drug Release sponges containing progesterone 12 days before mating. Rams wearing a harness carrying a coloured crayon are left with the ewes overnight 140 days before the planned delivery. Ewes not marked are regarded as not being pregnant. 14-17 days later, the rams are again exposed to the ewes, carrying a different coloured crayon. Ewes marked a $2^{\text {nd }}$ time are regarded as not having been mated during the first encounter, and are excluded from the flock. At between 40 and 50 days gestation, the ewes undergo a veterinary assessment to ensure that they are healthy and an Ultrasound (U/S) to confirm the pregnancy and identify singletons, twins and other multiples. In general, triplets and quadruplets are not utilised for research procedures, although such lambs may sometimes be used as normal controls. Prior to surgery, they are acclimatised to a limited supply of sheep nuts. Each ewe is weighed on the farm shortly before transport.

\section{Transport}

In general, we attempt to keep the ewes in the Laboratory environment for as short a time as possible. They are, therefore, transported to the Laboratory (lab) in a covered utility vehicle 24-72 hours prior to the planned day of surgery. They are housed in open pens with ewes being fasted being segregated by open mesh fencing. No ewe is ever left on her own. The concrete floors are covered with a layer of shavings derived from untreated pine. They are allowed free access to Lucerne hay and limited amounts of sheep nuts.

\section{Pre-op fasting}

Twenty-four hours preoperatively, the food is removed, although they are allowed free access to water. This differs from the approach most commonly described by most researchers using ovine models, with fasting of up to 48 hours being common [4,5]. However, we have found that sheep starved for prolonged periods tend to attempt to eat their bedding, and even if they are denied water for up to 48 hours, 


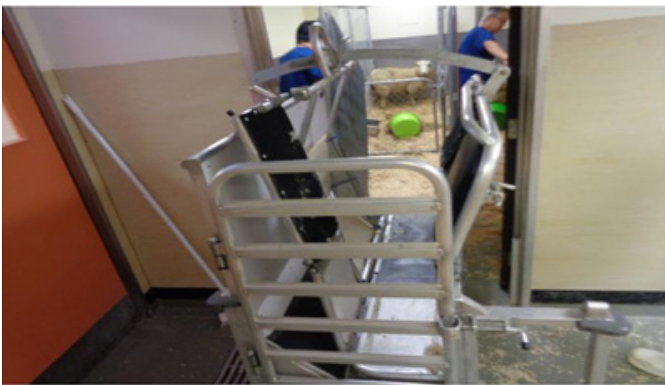

Figure 1: Tilting trolley in place, ready to load sheep.

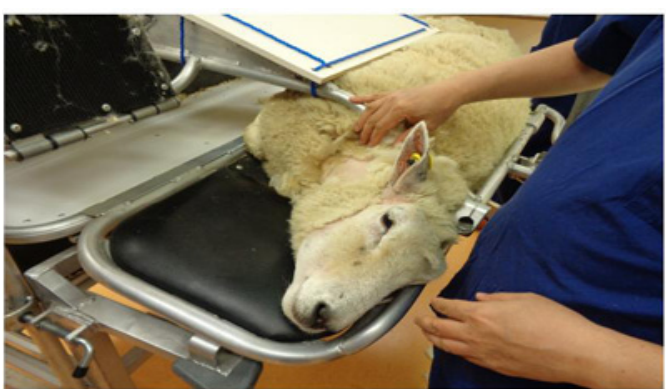

Figure 2: Sheep tilted. R neck shaved prior to injecting induction drugs.

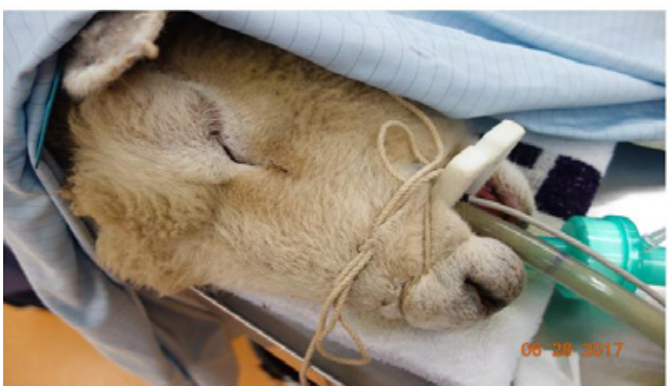

Figure 3: The intubated sheep. The ETT has been tied in with string and connected to a filter. The white bite block is seen protruding from the side of the mouth with the OG tube with the black line marking the length required to reach the stomach. The grey lead is the pulse oximeter which has been applied to the side of the mouth.

their rumen still is full of fluid. Sheep scheduled for surgery are kept in a separate cage, but are always able to see other sheep.

\section{Induction}

Approximately 30 minutes prior to the planned procedure, meloxicam $20 \mathrm{mg} / \mathrm{ml}(1 \mathrm{mg} / \mathrm{kg})$ is administered by subcutaneous injection as a post-operative analgesic. The ewe is transported from the pen to the operating theatre in a mobile tilting cage (Figure 1). The sheep is encouraged up a ramp and held fast in a grip. She is wheeled into the operating theatre, positioned next to the operating table and tilted on to her left side. The flap holding her head is released and the neck over the jugular vein is clipped (Figure 2). The jugular vein is easily demonstrated by obstructing it just above the clavicle, immediately medial to the sternomastoid. The jugular vein is cannulated with an 18 Gauge needle and the ewe is injected with a ketamine $(10 \mathrm{mg} / \mathrm{kg}) /$ diazepam $(0.5 \mathrm{mg} / \mathrm{kg})$ mixture. Within 2 minutes, the grip can be released and the ewe rolled over on to the

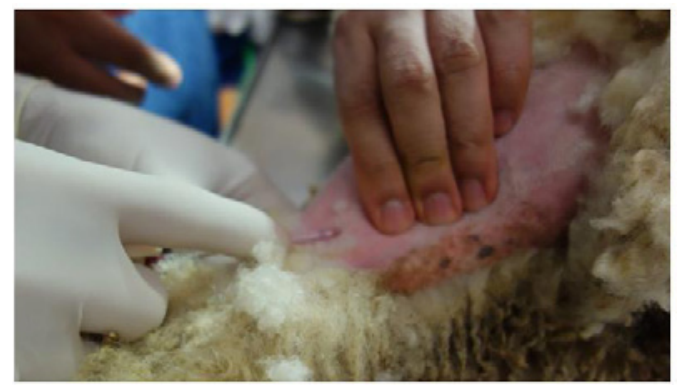

Figure 4: The $L$ hind limb just above the ankle has been clipped. The lateral tarsal vein is being cannulated.

operating table. The tongue is grasped with a gauze swab and pulled out over the lower jaw, taking care to avoid lacerating it on the teeth. Intubation is performed using a long straight-blade laryngoscope. In most cases a $7 \mathrm{~mm}$ Endotracheal Tube (ETT) with a low-pressure balloon is used. A plastic bite-block is passed over the end of ETT and the tube is tied to the upper jaw with string, with the ties passing around the both the upper and lower jaw, so the ewe cannot open or close her mouth. The ETT is connected to an anaesthetic machine and anaesthesia is maintained using a nitrous oxide/oxygen mixture (both $21 / \mathrm{min}$ ) and 1-2\% isoflurane (Figure 3). Mechanical ventilation is only used if the ewe is breath-holding or if the plane of anaesthesia needs to be deepened. Amoxycillin $(15 \mathrm{mg} / \mathrm{kg})$ is administered by intramuscular injection into the supraspinatus muscle. The scapula deep to the muscle ensures that no vital organs are injured. An OroGastric (OG) tube is passed to drain the stomach of fluid and gas. We use a $2 \mathrm{~cm}$ polyethylene tube with $1 \mathrm{~cm}$ side-holes cut in the gastric end. The tube is kept frozen between uses. Care must be taken when passing the tube, as excessive force can result in an oesophageal tear, which is usually lethal. If the tube thaws before it is reaches an adequate position, it is removed, washed and re-frozen.

The ewe's legs are tied to the edge of the table, using padded ropes. The lateral surface of the $\mathrm{L}$ hind limb is clipped and a $20 \mathrm{G}$ Intravenous (IV) cannula is placed in the lateral tarsal vein. This allows the delivery of 1 litre of Normal saline and 5\% Dextrose solution during the procedure.

\section{Preparation of surgical site}

The L side of the abdomen is initially clipped using an electrical shearer's handset. The area extends from the costal margin to a point well caudal of the iliac crest and from the spine down to the ventral surface of the abdomen. This also includes the upper part of the $\mathrm{L}$

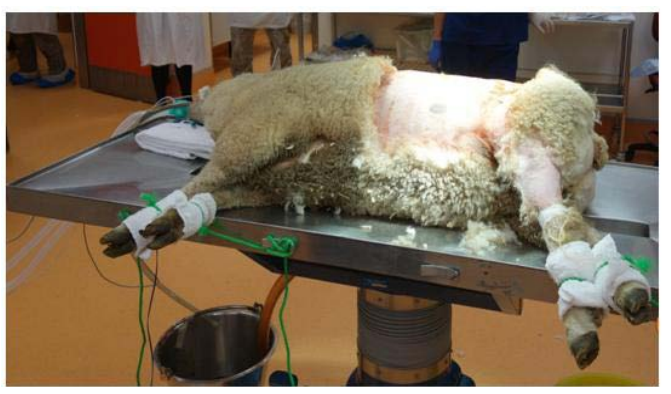

Figure 5: Ewe immediately before "prepping" the surgical site. Note the padded ties that lock all 4 limbs in place. 


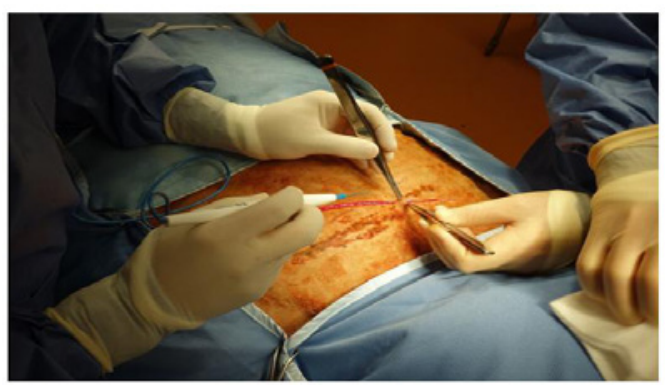

Figure 6: The skin incision has been made. The operating surgeon is standing at the ventral side of the ewe. The assistant's $L$ hand obscures the iliac crest. The transverse discolored patch is the remains of a cake of lanolin that lay on the skin under the wool.

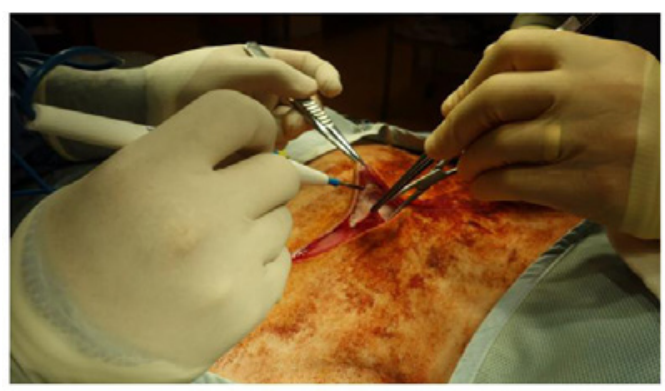

Figure 7: Panniculus carnosi is being opened. The subcutaneous fat can be seen. The surgeon is standing on ventral side of the ewe.

hind leg. Fine clippers are then used to complete the shaving. If the clipper blades become clogged with lanolin, then they are sprayed with $70 \%$ alcohol. When this technique was first developed, the area of the incision was further cleaned using a depilating cream such as "Nair" . We have abandoned this, as it adds expense for no reduction of wound problems (Figure 5). The shaved area is then cleaned with aqueous povidone-iodine solution. The area is then draped with the same sterile drapes as those used for human surgeries. We have found it useful to suture the drapes in place with some 3-0 nylon sutures, to prevent them slipping during the procedure. The operating table is metal, and the diathermy pad is simply applied to the underside of the table. For the initial stages of the dissection we set the diathermy at 50 for both cutting and coagulation and on "Blend 3".

\section{Abdominal incision}

For the first procedure, a muscle-splitting incision is used. If a muscle-cutting incision is used, the muscle layers are so thin that the sutures cut out when closure is attempted. The skin incision commences cephalad and slightly ventral to the iliac crest and continues ventrally and cephalad at an angle of approximately 30 degrees to the transverse plane (Figure 6). Immediately deep to the skin, panniculus carnosi is divided in the line of the incision, using either cutting or coagulating current. Individual bleeding vessels are controlled with coagulating current (Figure 7).

Deep to panniculus carnosi, there is usually a layer of fat that can be easily divided in the line of the incision. There are often moderately large vessels that need to be identified and coagulated before being divided.

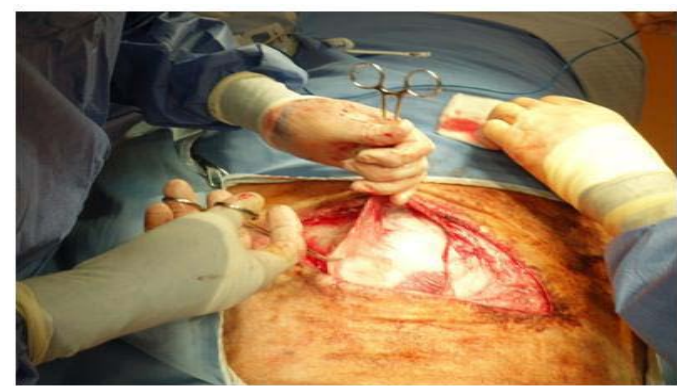

Figure 8: The ventral side of external oblique is being lifted vertically, to allow this layer to be dissected off the external surface of rectus sheath.

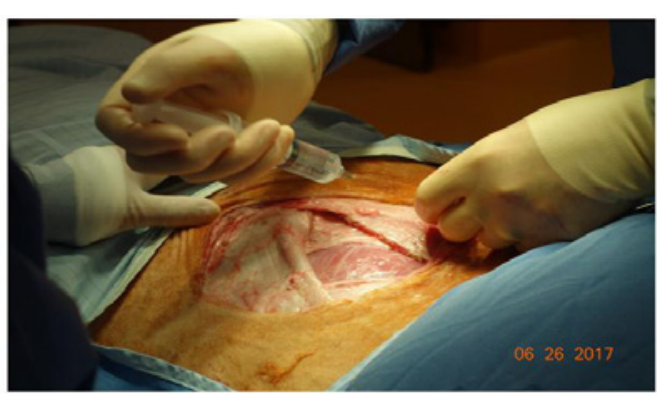

Figure 9: External oblique and internal oblique have been opened. One of the lumbar nerves is about to be injected with $0.25 \%$ bupivacaine.

External oblique is then divided in the line of its fibres. There is usually a transition between the aponeurosis and the muscle belly in the region immediately deep to the line of the incision. It is best to divide the aponeurosis first, extending the incision deep to the hind leg and then lifting the muscle belly between 2 fingers, continue the incision cephalad up to the costal margin. There is usually a vein approximately $10-15 \mathrm{~cm}$ cephalad from the beginning of the muscle belly that needs to be coagulated before it is divided. Clamps applied to the ventral cut edge of the muscle allow this to be lifted so that external oblique can be dissected off the anterior surface of rectus sheath, giving a greater length available for the incision in internal oblique (Figure 8).

Internal oblique is then opened in the line of its fibres from the dorsal end of the incision down to the rectus sheath. It is sometimes necessary to open the rectus sheath to get sufficient exposure. Before doing so, however, it is usually possible to reflect the external oblique fascia off the internal oblique, to obtain better exposure (Figure 8).

Immediately deep to the internal oblique, segmental nerves can be seen lying on transversus abdominus. These are followed to the point where they disappear under Internal oblique and each nerve has $2-3 \mathrm{ml}$ of $0.25 \%$ bupivacaine injected around it, for perioperative and post-operative pain relief (Figure 9). Any remaining bupivacaine is injected subcutaneously in the cephalad and dorsal regions of the wound.

Transversus is then split in the centre of the incision and the peritoneum opened. The incision is made between 2 of the lumbar nerves that travel parallel to the muscle fibres, far enough away from the nerves to allow closure of the incision without trapping the nerves (Figure 10). The incision is initially extended dorsally and cephalad, 


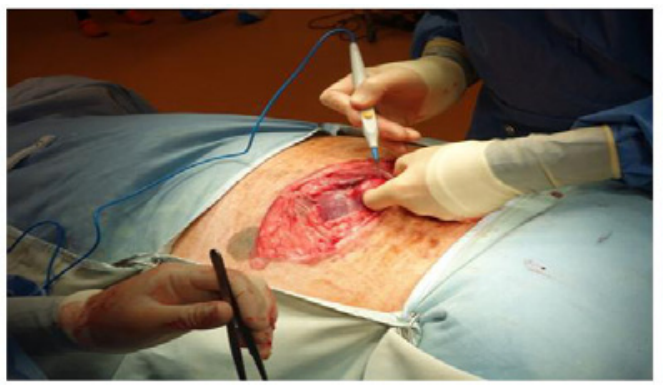

Figure 10: Transversus has been opened. A finger has been inserted into the peritoneal cavity and the incision is being extended cephalad and dorsally. A lumbar nerve is seen just to the $L$ of the finger that has been inserted into the peritoneal cavity.

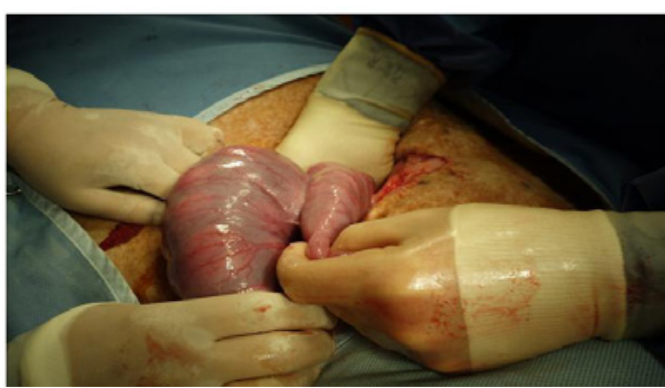

Figure 11: Delivering the uterus. The surgeon is standing on the side of the ewe's spine, with the $\mathrm{R}$ hand deep in the abdomen and gentle traction being applied to the horn of the $L$ the uterus. In this case, the $L$ horn was empty and there were twins in the $\mathrm{R}$ horn, something that occurs in about $10 \%$ of ovine pregnancies.

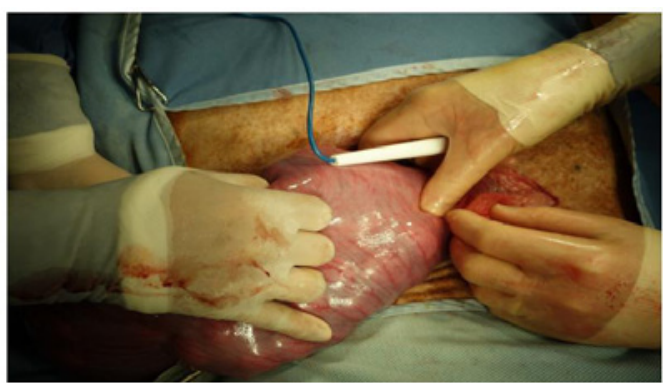

Figure 12: The lamb at the vaginal end of the uterus is being stabilised by the surgeon on the $L$ side of the picture. The caudal end of the lamb is under the diathermy stick.

deep to both internal oblique and external oblique. Care must be taken at the upper extent of this incision, as the ovarian vessels are at risk at the uppermost end of the incision. Once that is complete, the caudad portion of the incision is completed, deep to the hind limb.

\section{Delivery of the uterus}

As soon as the abdomen is opened, the position of the OG tube in the stomach is confirmed. If the stomach is full of gas, then the solid food in the tube can usually be dislodged by gripping the tube to wrap the gastric wall around the tube and squeezing gently. The senior author prefers to stand on the dorsal side of the ewe. This allows him to pass his right hand into the abdomen identify the fallopian tube end of both uterine horns. With the Right (R) hand under the body

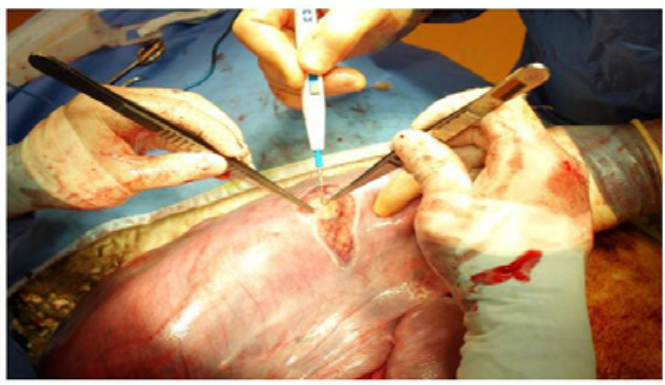

Figure 13: Opening the uterus. The uterine muscle has been opened between the uterine vessels, a series of parallel red lines best seen to the $L$ of the picture. The darker fetal vessels are being coagulated prior to opening the amnion. The head of the lamb is being stabilised by the surgeon's R hand to the $\mathrm{R}$ of the picture.

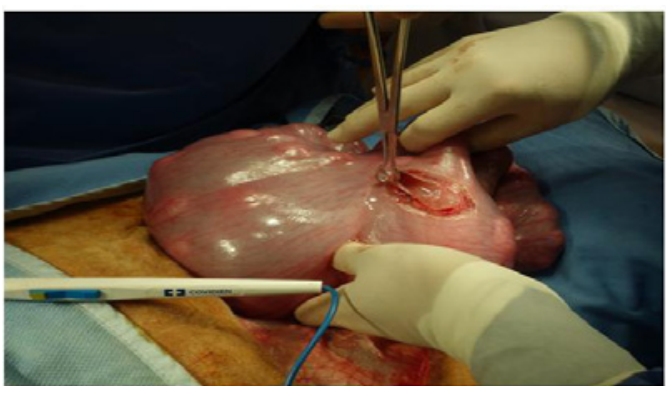

Figure 14: The uterus has been opened and the Babcock forceps has been applied to both the amnion and the uterine wall. The lamb can be seen inside the uterus. The lamb's head is still being stabilised by the surgeon's R hand at the top of the picture.

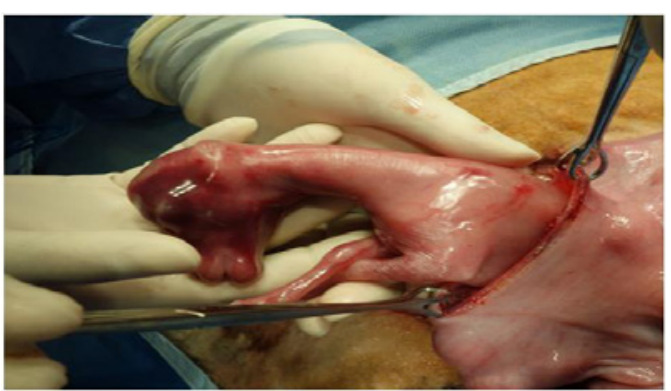

Figure 15: Cephalic end of a 75-day gestation fetal lamb delivered for a $\mathrm{L}$ thoracotomy

of the $\mathrm{R}$ uterine horn and the $\mathrm{L}$ hand providing gentle traction on the fallopian tube end of the uterus, it is usually possible to deliver both horns out of the wound (Figure 11). The number and position of the lambs can be confirmed by gentle palpation through the thin uterine wall. Lambs of less than 75-80 days gestation can be difficult to identify because of the large volumes of amniotic fluid. In general, if twins are to both undergo a procedure, the $\mathrm{R}$ twin is operated upon first.

\section{Uterine incisions}

The lamb is identified and positioned against the uterine wall. We generally stabilise the lamb by looping a finger and thumb around the lamb's neck, with the lamb still inside the uterus (Figure 12). In lambs less than 90-100 days gestation, the procedure is accomplished by 


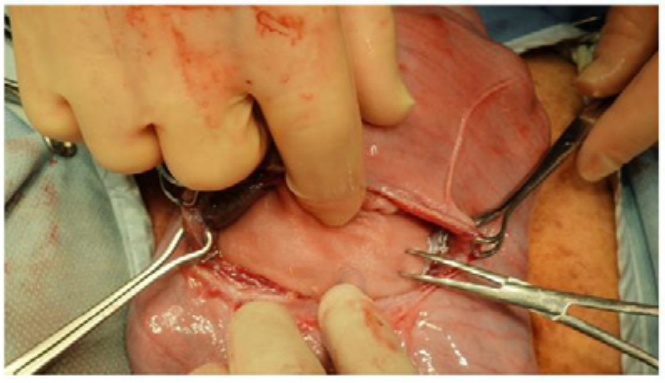

Figure 16: $L$ subcostal region of a 110-gestation fetal lamb exposed to repai a diaphragmatic hernia.

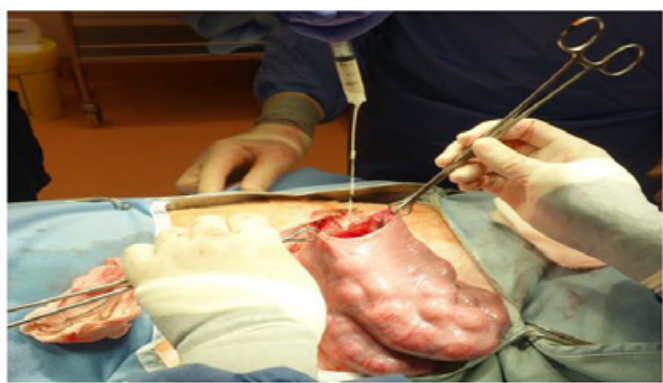

Figure 17: Injection of intra-amniotic antibiotics immediately before uterine closure.

delivering the appropriate pole of the lamb through the hysterotomy. In lambs older than 100 days gestation, an incision is made over the proposed site of the fetal procedure. With the lamb appropriately positioned, a transverse incision is made in the uterine wall between branches of the uterine vessels, using the cautery set at 20 for both cutting and coagulation. It is important to avoid placing the incision too close to a cotyledon, as they tend to prolapse through the hysterotomy. This is done cautiously with any fetal vessels (recognised by the darker colour of the blood within them) being cauterised before division to avoid fetal blood loss (Figure 13). The incision is deepened layer by layer until the amnion is opened. Care is taken to avoid excessive loss of amniotic fluid, although small losses are usually tolerated well. The ends and the sides of the incision are grasped in Babcock forceps, taking care to ensure that the amnion is included in the "bite" (Figure 14). In small lambs, the appropriate pole of the lamb is delivered through the hysterotomy and the wound is held around the lamb's body to minimise amniotic fluid losses (Figure 15). In lambs 100 days gestation or older, the appropriate part of the lamb is held against the incision and the fetal procedure is performed through the uterine window (Figure 16). We have successfully created Obstructive Uropathies (OU) in lambs as young as 50 days gestation, with acceptable fetal losses. In small lambs, especially, it is critical that care be taken to avoid compression of the umbilical vessels against the edge of the hysterotomy during the fetal procedure.

\section{Uterine closure}

Once the fetal procedure has been completed, the fetus is returned to the uterus, if it has been partially delivered. $500 \mathrm{mg}(2 \mathrm{ml})$ of amoxicillin is introduced into the amniotic cavity through the hysterotomy (Figure 17). The uterus is then closed with a running 3-0 absorbable suture, taking care to include the amnion in each

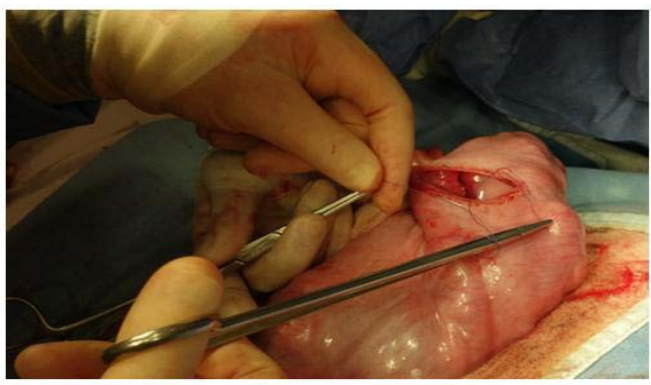

Figure 18: Beginning the first layer of uterine closure. The $1^{\text {st }}$ suture has been placed, taking care to include both the amnion and the muscle and is being tied.

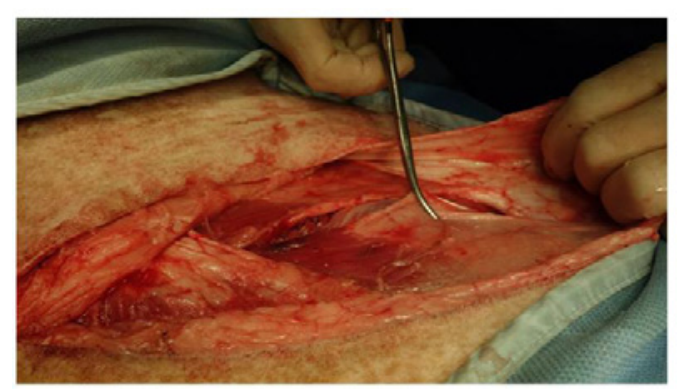

Figure 19: Closure of internal oblique. The aponeurosis at the ventral end of the incision has been grasped in forceps. The closure begins at the ventral end of the incision and continues dorsally.

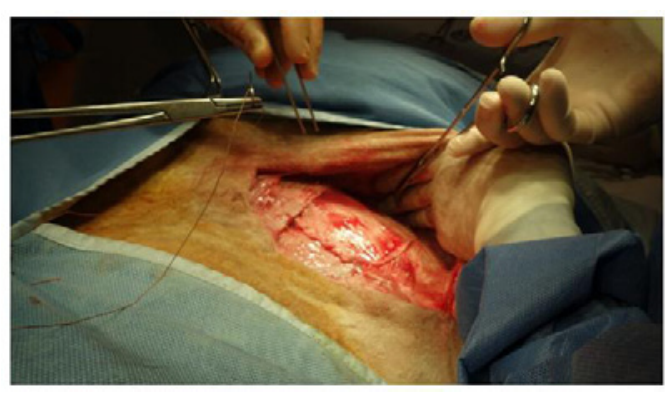

Figure 20: Closure of external oblique. The skin and subcutaneous tissue at the caudal end of the wound is being retracted. The haemostat is grasping the caudal end of the wound and the $1^{\text {st }}$ suture is about to be placed. Care needs to be taken at this point, as the femoral vessels are close to the end of the incision.

bite (Figure 18). After the $1^{\text {st }}$ layer is completed, an Aberdeen knot is formed and a $2^{\text {nd }}$ layer of sutures is placed, imbricating the $1^{\text {st }}$ layer. These sutures are seromuscular sutures, placed parallel to the uterine vessels, inverting and covering the $1^{\text {st }}$ layer.

\section{Abdominal closure}

Transversus is closed with a running 2-0 absorbable suture. It is best to begin at the caudal end of the incision and work towards the cephalic end. Good retraction is needed to provide exposure and it is important to take care placing the initial sutures as a carless movement of the needle risks damage to the femoral vessels lying on the medial surface of the thigh.

Internal oblique is closed with a running 0 absorbable suture (Figure 19), as is external oblique. It is best to begin the closure of 


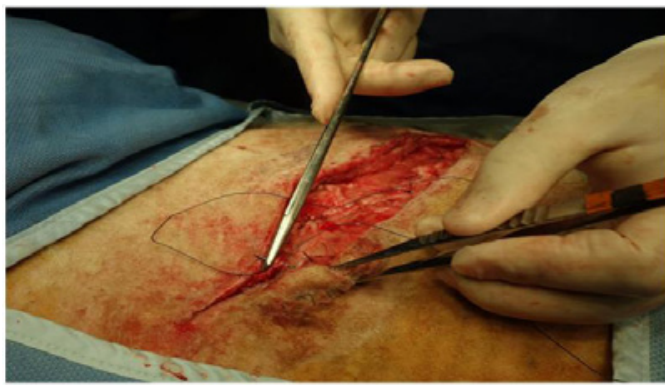

Figure 21: Beginning of the closure of panniculus carnosi. This is commenced at the dorsal end of the incision and proceeds ventrally. In our operating team, various members of the team take different roles as surgeon or assistant, dictated as much as anything by the side of the table on which they are standing and the ease with which they can perform that task from where they are standing.

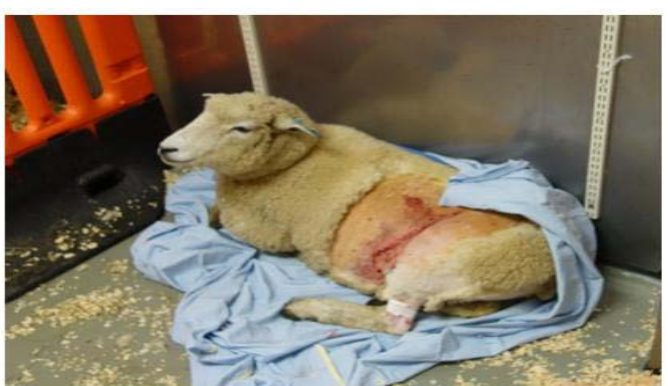

Figure 22: Ewe immediately after being returned to the recovery area.

external oblique at the caudal end of the wound. Again, care is needed at the beginning of the closure, as the femoral vessels can easily be injured by the needle point as the needle is passed through the aponeurosis (Figure 20).

The subcutaneous fat is approximated with 0 or $2-0$ absorbable sutures. Panniculus carnosi is closed with a running 2-0 absorbable suture. Figure 21 for the initial incision a taper needle can be used. Fir subsequent procedures, a cutting needle is best. The skin is closed using a 3-0 monofilament suture, either absorbable or nonabsorbable. We have found that if a braided suture is used for the skin, there is a significant risk of superficial wound infections. Conversely, monofilament sutures can be safely left in place until the next procedure. The wound is then sprayed with "Opsite ${ }^{\infty}$ " spray to discourage flies from attacking the fresh wound.

\section{Emergence and post-op care}

The nitrous oxide and isoflurane is turned off when the closure of the panniculus carnosi is commenced. The $\mathrm{O}-\mathrm{G}$ tube is removed as the ewe begins to emerge from the anaesthetic. The ETT is only removed once the ewe begins chewing. The ties around her legs are removed at this time and she is then transferred on to one of the drapes covering a trolley for transfer back to the recovery area. She is placed supported on her brisket, leaning against the wall. In most cases, she is able to hold her head up at this time (Figure 22). As soon as she is walking freely, she is allowed to join the rest of the flock (Figure 23).

\section{Subsequent procedures}

For procedures carried out within a week of the first procedure, the muscle-splitting incision is simply re-opened. Such procedures

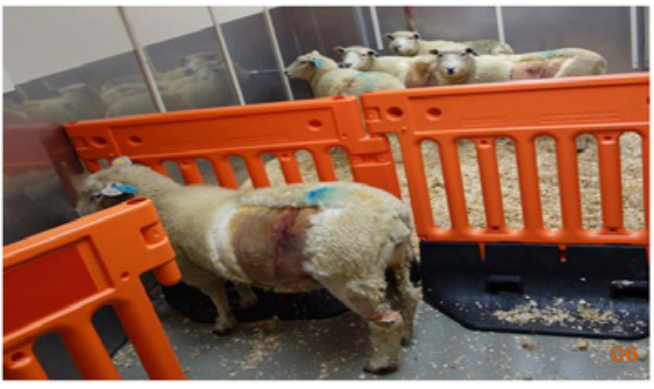

Figure 23: Thirty minutes later, she is ready to join the four ewes that had been operated upon earlier that day.

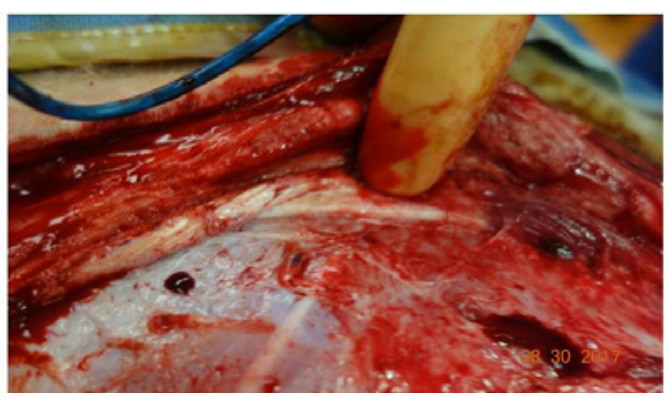

Figure 24: Subsequent procedure. The skin, panniculus carnosi, subcutaneous tissue, external oblique and internal oblique have been divided in the line of the incision. The finger is retracting internal oblique and indicating the lumbar nerve that is to be injected with bupivacaine.

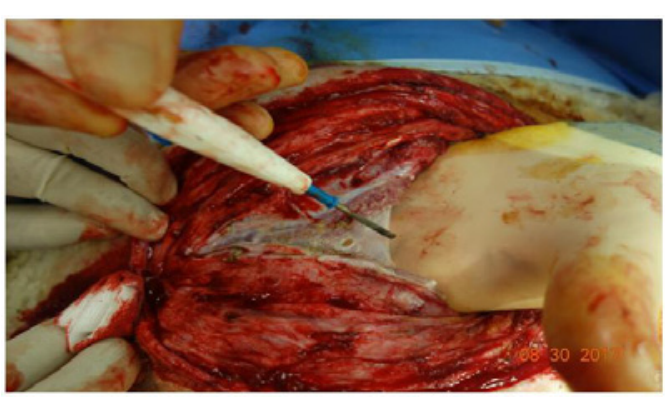

Figure 25: Subsequent procedure. Transversus has been opened and hand has been inserted as the ventral extent of the wound is being opened A lumbar nerve is visible at the ventral end of the incision in internal oblique.

usually result in moderate amounts of bleeding from the fresh granulation tissue in each layer. For procedures carried out after 10 days, a muscle-cutting incision is used.

After the ewe is anaesthetised, and IV access is obtained, any retained skin sutures are removed and the operative area is again clipped. The site is prepped and draped as outlined above. The skin incision is reopened using a scalpel and panniculus carnosi is divided in the line of the incision using the cautery. The subcutaneous fat is divided in the line of the incision as is external oblique. The incision in internal oblique is usually almost parallel to the original skin incision and can be reopened along this line. The cephalad flap is raised to allow identification of the lumbar nerves which are injected with $0.25 \%$ bupivacaine as outlined above (Figure 24). Any remaining bupivacaine is again injected into the subcutaneous layers cephalad and dorsal to the incision. Transversus is then opened in the line of 
the incision near the centre of the wound. Once the peritoneum is opened, the wound is extended ventrally initially and then dorsally (Figure 25). Again, care is needed at the dorsal extent of the wound to avoid damage to the ovarian vessels. Adhesions in the region of the previous incision in transversus are rare, but if present they are usually caudal to the wound. If necessary, they can be easily taken down to allow the uterus to be delivered.

After the fetal procedure is completed, the wound is closed in layers as outlined above. Because of the scarring associated with the initial procedure, the sutures do not cut through and the closure is invariably sound.

\section{Results and Discussion}

The senior author developed this technique in 1980, together with Dr John Schofield, a veterinary surgeon. It was first reported in $1984[7,8]$ At that time, the induction agent was ketamine. Initially, the skin was routinely closed with 3-0 monofilament nylon, which was removed at 10-14 days. Occasionally the sutures were forgotten and it became apparent that even if a nylon suture is used, there are no untoward effects if the sutures are left in place until the next procedure. However, we now generally use 3-0 monofilament absorbable sutures. As noted above, the incidence of superficial skin infections is relatively high if braided sutures are used. In general, these cause no systemic harm, but the risk of fetal losses increases if the infection is still present at the time of a second procedure, especially if some sort of implant is used. This approach has enabled us to ligate the fetal urethra as early as 50 days gestation [9] and repair a diaphragmatic hernia as late as 123 days gestation [7].

Subcutaneous seromata are commonly encountered during second operations performed 4-6 weeks after the initial operation. They usually lie deep to the subcutaneous fat on the surface of external oblique. They have never been found at term. They have no adverse effect that we have found.

It is also possible to use this incision to deliver term lambs. In un-operated controls, if the ewe is to be euthanased after the delivery, then a muscle-cutting incision is used, simply dividing the muscles in the line of the usual skin incision, as closure is not required. If the ewe were to be required to feed her lambs after delivery, then a musclesplitting incision would be used. If the lambs are to be delivered after a previous intervention, then the original incision is simply re-opened using the muscle-cutting incision as outlined above for subsequent procedures.

\section{Conclusion}

The left flank approach is a safe and flexible approach through which to approach the ovine fetus.

\section{References}

1. Schinekel PG, Ferguson KA. Skin transplantation in the foetal lamb. Aust $J$ Biol. 1953; 6: 533-546.

2. Beck $A D$. Intrauterine renal surgery: techniques for exposing the fetal kidney during the last two-thirds of gestation. Invest Urol. 1970; 8: 182-187.

3. De Lorimier AA, Tierney DF, Parker HR. Hypoplastic lungs in fetal lambs with surgically produced diaphragmatic hernia. Surgery. 1967; 62: 12-17.

4. Tanagho EA. Surgically induced partial urinary obstruction in the fetal lamb. I. Technique. Invest Urol. 1972; 19-24.

5. Harrison MR, Jester JA, Ross NA. Correction of diaphragmatic hernia in utero. I. The model: Intrathoracic balloon produces fatal pulmonary hypoplasia. Surgery. 1980; 88: 174-182.

6. Burrington JD. Wound healing in the fetal lamb. J Pediatr Surg. 1971; 6: 523528.

7. Soper RT, Pringle KC, Scofield JC. Creation and repair of diaphragmatic hernia in the fetal lamb: Techniques and survival. J Pediatr Surg. 1984: 19; 33-40.

8. Pringle KC. Fetal lamb and fetal lamb lung growth following creation and repair of a diaphragmatic hernia. PW Nathanielsz, editors. In: Perinatology Press. Animal Models in Fetal Medicine. 1984; 4: 109-148.

9. Kitagawa H, Pringle KC, Zuccollo JM, Koike J, Nakada K, Moriya H, et al. Early fetal obstructive uropathy produces Potters syndrome in the lamb. J Pediat Surg. 2000; 35: 1549-1553.
Austin J Obstet Gynecol - Volume 4 Issue 2 - 2017

Submit your Manuscript | www.austinpublishing group.com

Pringle et al. ( ) All rights are reserved
Citation: Pringle KC, Seki Y and Kitagawa H. Surgical Access to the Ovine Fetus the Left Flank Approach. Austin J Obstet Gynecol. 2017; 4(2): 1076. 